\title{
The ALERT Algorithm: How to Simply Define a Period of Elevated Disease Incidence
}

\author{
Nicholas G. Reich¹, Derek Cummings², Martha Zorn¹, Ann-Christine Nyquist", Trish M. \\ Perl $^{5}$ and Lew J. Radonovich ${ }^{3}$
}

'Division of Biostatistics and Epidemiology, University of Massachusetts-Amherst, Amherst, MA, USA; ${ }^{2}$ Johns Hopkins Bloomberg School of Public Health, Baltimore, MD, USA; ${ }^{3}$ Veterans Health Administration, Gainesville, FL, USA; ${ }^{4} \mathrm{Children's} \mathrm{Hospital} \mathrm{Colorado,}$ Aurora, CO, USA; 5 Johns Hopkins University School of Medicine, Baltimore, MD, USA

\section{Objective}

Our objective was to develop a simple, easy-to-use algorithm to predict the onset of a period of elevated influenza incidence in a community using surveillance data.

\section{Introduction}

Despite the number of infections, hospitalizations, and deaths from influenza each year, developing the ability to predict the timing of these outbreaks has remained elusive. Public health practitioners have lacked a reliable, easy-to-implement method for predicting the onset of a period of elevated influenza incidence in a community. We - a team of statisticians, epidemiologists, and clinicians - have developed a model to help public health practitioners develop simple, adaptable, data-driven rules to define a period of increased disease incidence in a given location. We call this method the Above Local Elevated Respiratory illness Threshold (ALERT) algorithm. The ALERT algorithm is a simple method that defines a period of elevated disease incidence in a community or hospital that systematically collects surveillance data on a particular disease.

\section{Methods}

A user of the system, such as a hospital epidemiologist or surveillance system administrator, specifies the percentage of cases they want the ALERT period to capture.

The ALERT algorithm then uses historical surveillance data to determine an optimal threshold that is used to determine the beginning and end times of the ALERT period. For example, at the beginning of an influenza season, when the number of observed cases crosses the threshold, the ALERT period begins. And, once this threshold is crossed again (on the epidemic's downswing), the ALERT period ends. For a range of possible thresholds, the ALERT algorithm calculates the percentages of cases observed within the ALERT period defined by each threshold. Using a variety of numerical and visual summaries, the user may choose the best threshold for their needs or use the one suggested by ALERT algorithm.

\section{Results}

Using simple and user-adaptable criteria to determine the best threshold, the ALERT algorithm successfully predicted the start of periods of elevated disease incidence in multiple hospital settings in the 2011-12 and 2012-13 influenza seasons. We tested the algorithm in pediatric and primary care hospitals that reside in different geographic regions of the United States.

\section{Conclusions}

By clearly and simply defining a period of increased risk, the ALERT algorithm allows administrators to plan proactively and enables fast response to emerging outbreaks in healthcare settings. It therefore could aid in efforts to prevent excess cases of disease. The ALERT algorithm may be run on any user's computer via a simple spreadsheet. A web-based application that implements the ALERT algorithm is currently under development.

\section{Keywords}

influenza; outbreak detection; public health practice; surveillance

\section{Acknowledgments}

NGR was funded by the ResPECT study (clinicaltrials.gov, ID: NCT01249625) through an interagency agreement between the Centers for Disease Control and the United States Department of Veterans Affairs (CDC IAA 09FED905876). The findings and conclusions in this manuscript are those of the authors and do not necessarily represent the views of the Centers for Disease Control and Prevention. The funders had no role in study design, data collection and analysis, decision to publish, or preparation of the manuscript.

\author{
*Nicholas G. Reich \\ E-mail: nick@schoolph.umass.edu
}

